\title{
Insight into Indole Interactions from Alkali Metal Chloride Effects on a Tryptophan Zipper $\beta$-Hairpin Peptide
}

\author{
Christopher E. Dempsey* ${ }^{\star \dagger}$ and Philip E. Mason ${ }^{\ddagger}$ \\ Biochemistry Department and Centre for Molecular Recognition, School of Medical Sciences, University \\ Walk, Bristol BS8 1TD, U.K.*, and Department of Food Science, Stocking Hall, Cornell University, Ithaca, \\ N.Y. 14853
}

This supporting information contains representative spectroscopic data on the conformational stability of trpzip1, MrH4a and alahel (E7) in alkali metal salt solutions, together with graphical analyses for the determination of the $m$ values listed in Table 2 of the Communication. The reference numbers in this Supporting Information refer to references in the Communication.

\section{Brief experimental details:}

Peptide synthesis and purification: Peptides were synthesised by Dr. Graham Bloomberg of the Bristol University Centre for Molecular Recognition, purified by HPLC, and confirmed to be at least $96 \%$ pure by analytical HPLC, and to have the predicted $m / e$ ratio by mass spectrometry. ${ }^{3}$

Spectroscopic measurement: Measurements were made in a buffer composed of $10 \mathrm{mM}$ potassium phosphate adjusted to $\mathrm{pH} 7.0$ or $\mathrm{pH} 2.5$. Peptide concentrations were determined using either the Trp absorbance of the trpzip peptides $\left(\varepsilon_{560}=5600 \mathrm{M}^{-1} \mathrm{~cm}^{-1}\right)$ or the Tyr absorbance of alahel $\left(\varepsilon_{275}=1450 \mathrm{M}^{-1} \mathrm{~cm}^{-1}\right)$. The concentration of $\mathrm{MrH} 4 \mathrm{a}$ was determined by integration of HPLC peak intensities in comparison with peptides of known concentration. Circular dichroism spectra were obtained in cuvettes of $1 \mathrm{~mm}, 2 \mathrm{~mm}$ or $10 \mathrm{~mm}$ path length using a Jobin-Yvon CD6 spectrapolarimeter, with the temperature of the cuvette holder maintained using a Haake circulating water bath. Accurate temperatures were obtained by direct measurement within samples using a Hanna H198801 thermocouple thermometer.

Analysis of Spectroscopic Data: Data were measured in molecular ellipticity mode. In all cases a relevant blank spectrum was subtracted. Sufficient scans were run such that accurate measurement of ellipticity values used for determining structural content could be obtained without spectral smoothing. The proportion of the folded form of each peptide ( $f_{H}$, the fraction helix in alahel; $f_{\mathrm{B}}$, the fraction of $\beta$-hairpin in trpzip peptides and MrH4a) were determined as described in ref 3 . The relevant equations are:

$$
\mathrm{f}_{\mathrm{B}(\mathrm{tr} z z i p)}=\frac{\theta_{227}-\theta_{\mathrm{u}}}{\theta_{\max }-\theta_{\mathrm{u}}}
$$

where $\theta_{\max }=921,000 \mathrm{deg} \mathrm{cm}^{2} \mathrm{~mol}^{-1}$ and $\theta_{\mathrm{u}}=29,400 \mathrm{deg} \mathrm{cm}^{2} \mathrm{~mol}^{-1}{ }^{1,3}$ Values of $\mathrm{f}_{\mathrm{B}}$ for MrH4a were determined from $\theta_{222}$ based on the observation of Dyer et al. that $\mathrm{f}_{\mathrm{B}}$ for $\mathrm{MrH} 4 \mathrm{a}$ at $31{ }^{\circ} \mathrm{C}, \mathrm{pH}$ 7.5 is $0.31 .^{4}$ Using the C-terminal_N-acetyl-hexapeptide of MrH4a as an analog of the unfolded stateCD spectrum, we estimated that $30 \%$ of the value of $\theta_{222}$ for $\mathrm{MrH} 4 \mathrm{a}$ at $31^{\circ} \mathrm{C}$ was retained in the unfolded state (not shown) The fraction of helix, $f_{H}$, for alahel (E7) was determined from:

$$
\mathrm{f}_{\mathrm{H}}=\frac{\theta_{222}-\theta_{\mathrm{r}}}{0.8125(-44000+250 \mathrm{~T})-\theta_{\mathrm{r}}}
$$

where $\mathrm{T}$ is the temperature in degrees Celsius, $\theta_{\mathrm{r}}$ is $\theta_{222}$ for the random coil form of the peptide $\left(\theta_{\mathrm{r}}=2200-53 \mathrm{~T}\right)$ (see ref. 3 for further explanation). 
Representative spectral data and graphical analyses for determination of $m$ values:

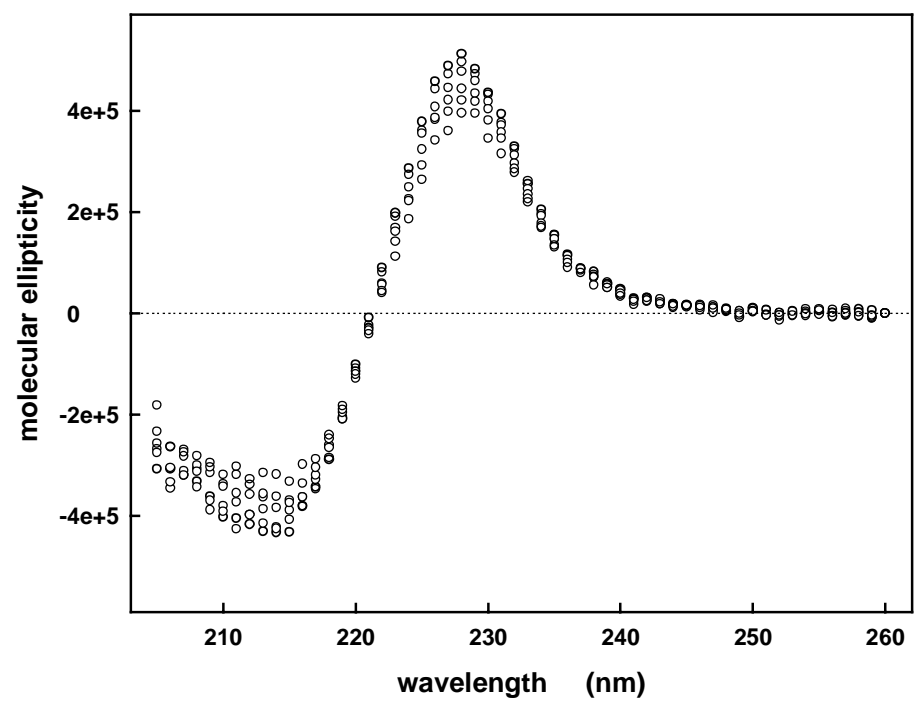

Figure 1. Rubidium chloride (RbCl)-dependence of the circular dichroism spectrum of trpzip1 $(10 \mu \mathrm{M})$ in $10 \mathrm{mM}$ potassium phosphate, $\mathrm{pH} 2.5$ at $42^{\circ} \mathrm{C}$. The $\mathrm{RbCl}$ concentrations are (top to bottom), $0,0.5,1,1.5$, 2 and $3 \mathrm{M}$.

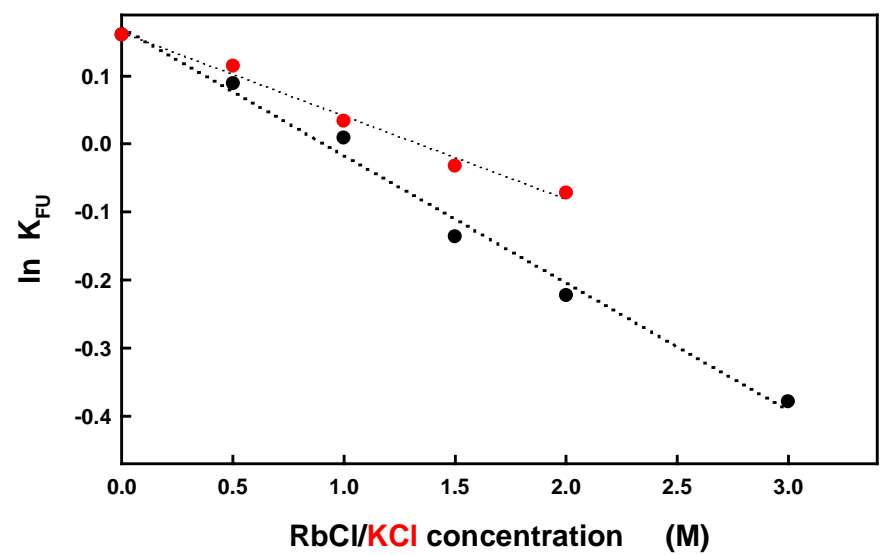

Figure 2. $\mathrm{RbCl}$-dependence and $\mathrm{KCl}$-dependence of the natural logarithm of $\mathrm{K}_{\mathrm{FU}}$, the equilibrium constant defining the ratio of folded and unfolded forms of trpzip1 $\left(42^{\circ} \mathrm{C}, 10 \mathrm{mM}\right.$ potassium phosphate, $\mathrm{pH}$ 2.5). The dotted lines are a linear regression fit to the data. The gradient is equal to $m / R T$ (see equation 1 in the communication). 

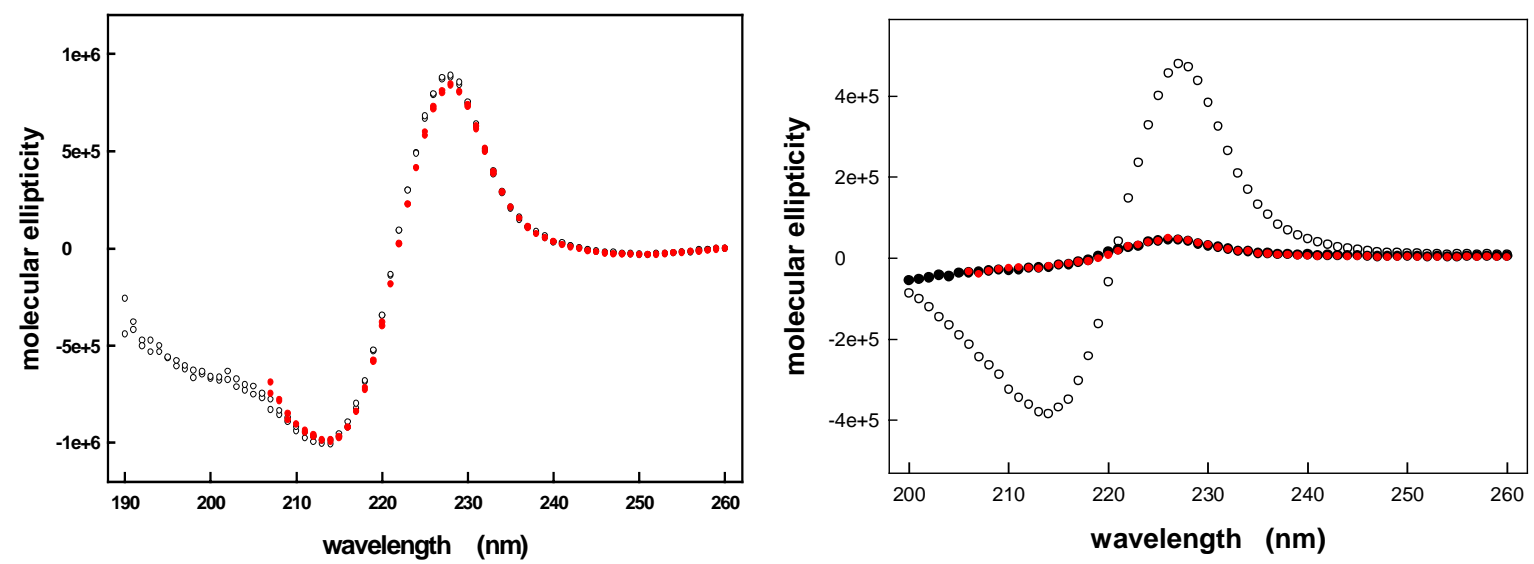

Figure 3. Left: $\mathrm{CD}$ spectrum of trpzip2 $(10 \mu \mathrm{M})$ in $10 \mathrm{mM}$ phosphate buffer, $\mathrm{pH} 2.5,20^{\circ} \mathrm{C}$ (open circles) and in the presence of $2 \mathrm{M} \mathrm{RbCl}$ (red circles). This peptide is considerably more stable than trpzip, ${ }^{1}$ and is almost completely folded at $20^{\circ} \mathrm{C}$ even at $\mathrm{pH} 2.5$ where the E5-K8 salt bridge is disrupted by protonation of the $\mathrm{E} 5$ side chain carboxyl. $\mathrm{RbCl}$ at a concentration of $2 \mathrm{M}$ has only a small effect on the maximum ellipticity, indicating that the salt doesn't significantly affect the CD signal from the Trp indoles of trpzips in the folded state. Right: CD spectrum of trpzip1 (buffer as above) at $42{ }^{\circ} \mathrm{C}$ (open circles; $20 \mu \mathrm{M}$ ), and of a peptide corresponding to the C-terminal pentapeptide of trpzip1 (i.e. Ac-KWTWK-NH${ }_{2}$ ) at $40 \mu \mathrm{M}$, in the absence (filled circles) and presence (red circles) of $2 \mathrm{M} \mathrm{RbCl}$. Note that the pentapeptide was measured at double the trpzip1 concentration so that the overall Trp concentration is equivalent in each sample; the molecular ellipticity values for the pentapeptide are effectively "trpzip1 equivalents", i.e. they are double the true molecular ellipticity values for this peptide. The very minor effects of $\mathrm{RbCl}$ on both the folded state signal arising from the indole-indole interaction (left panel) and an analog of the unfolded state (right panel), support the conclusion that the alkali metal salt effects on the CD spectrum of trpzip1 result from shifts of the folding equilibrium $\mathrm{K}_{\mathrm{FU}}$.

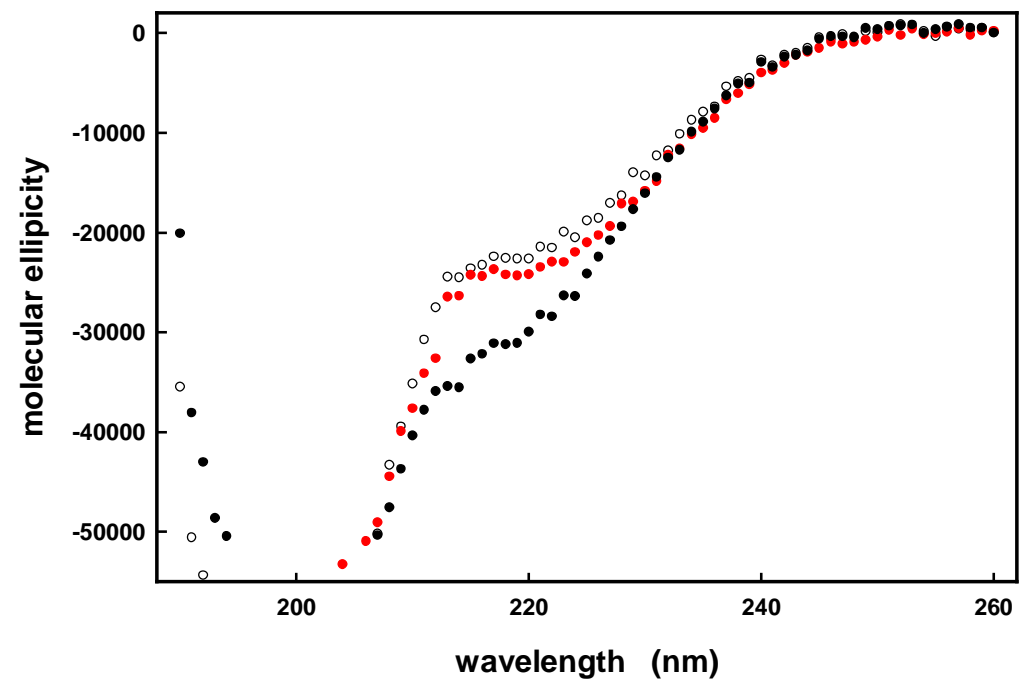

Figure 4. Circular dichroism spectra of MrH4a in $10 \mathrm{mM}$ phosphate, $\mathrm{pH} 7.0$ (black circles), $10 \mathrm{mM}$ phosphate, $\mathrm{pH} 2.5$ (open circles) and $10 \mathrm{mM}$ phosphate, $\mathrm{pH} 2.5$ containing $2 \mathrm{M} \mathrm{NaCl}$ (red circles). The effects of $2 \mathrm{M} \mathrm{LiCl}, \mathrm{KCl}$ or $\mathrm{RbCl}$ on the $\mathrm{MrH} 4 \mathrm{a}$ spectrum at $\mathrm{pH} 2.5$ were very similar to that shown for $2 \mathrm{M}$ $\mathrm{NaCl}$ (not shown), and each salt was assigned the same $m$ value $\left(-34 \mathrm{cal} \mathrm{mol}^{-1} \mathrm{M}^{-1}\right)$ calculated for $\mathrm{NaCl}$ on the folding equilibrium of $\mathrm{MrH} 4 \mathrm{a}$. 


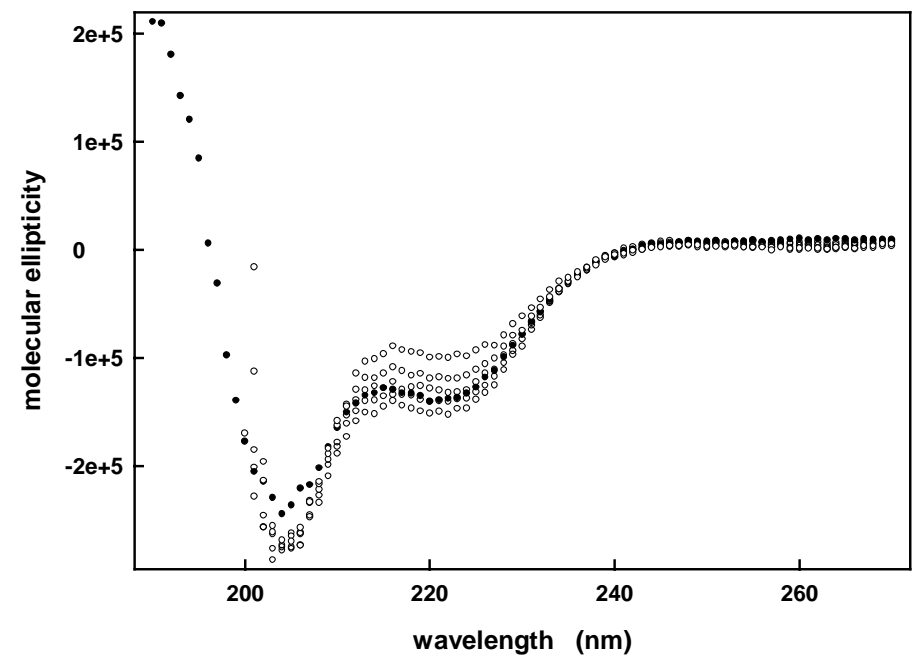

Figure 5. LiCl-concentration dependence of the CD spectrum of alahel $(E 7)(40 \mu \mathrm{M})$ in $10 \mathrm{mM}$ potassium phosphate, $\mathrm{pH} 7.0$ at $15^{\circ} \mathrm{C}$. The LiCl-free spectrum is highlighted with black symbols to illustrate the small stabilisation of the helical structure at $0.5 \mathrm{M} \mathrm{LiCl}$ due to screening of helix dipole charges. The open circles correspond to $\mathrm{LiCl}$ concentrations of $0.5,1,1.5,2$ and $3 \mathrm{M}$, $\mathrm{LiCl}$, bottom to top, respectively.

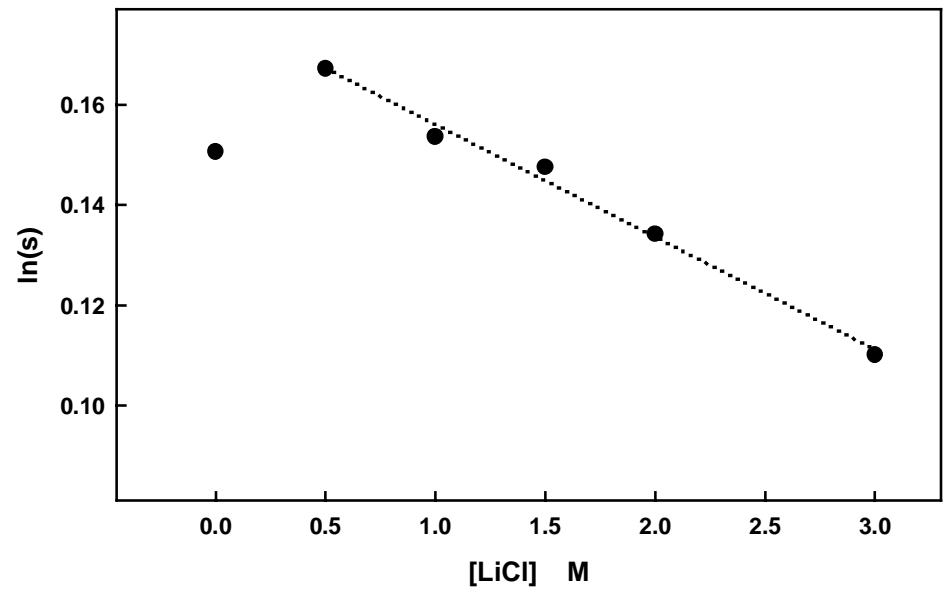

Figure 6. Variation of $\operatorname{In}(\mathrm{s})$, the helix propagation parameter (see equation S3), as a function of lithium chloride concentration. The dotted line is a linear regression fit of the data between $0.5 \mathrm{M} \mathrm{LiCl}$ and $3 \mathrm{M} \mathrm{LiCl}$. The salt-free point was omitted from the fit due to the small stabilisation of helical structure resulting from screening of helix dipole charges that is unrelated to electrolyte effects on hydrogen bonds (see Figure 5, and ref 3 of the communication).

As indicated in the communication, the $m$ values for electrolyte effects on the $\beta$-peptides are determined from equation 1, and are per mole of peptide. The $m$ values for alahel (E7) were determined using the Zimm-Bragg formalism as described in ref. 3, where the helix propagation parameter, $s$, is calculated from the value of $\mathrm{f}_{\mathrm{H}}$, the fraction of helix at each electrolyte concentration (equation S2). The $m$ value is then determined from equation S3 where $s_{0}$ is the helix propagation parameter in the absence of added electrolyte. Values of $m$ for helical peptides calculated using Zimm-Bragg s values are per residue.

$$
\ln s=\ln s_{0}-\frac{m[\text { solute }]}{\mathrm{RT}}
$$

